\title{
Numerical Study Of Three-Winged Passive Micromixer Based On Sar Principle
}

\author{
Monther Jomha ${ }^{1}$, Sedat Yayla ${ }^{2}$ \\ 1 Department of Mechanical, Faculty of Engineering, University of Van Yuzuncu Yil, \\ Van, Turkey \\ madarekna@gmail.com; syayla@yyu.edu.tr \\ 2 Head of Mechanical Department, Faculty of Engineering, University of Van Yuzuncu Yil, \\ Van, Turkey
}

\begin{abstract}
The passive three wings micromixer is presented according to the SAR principle. CFD numerically examined the mixer's efficiency for a wide range of Reynolds numbers up to 100. Three factors were used to test the efficiency of mixer performance. These three were the change in the fluids inlets velocity, connections with twisted angle, and a variable diameter obstacle to validate the submitted model. Besides, the pressure drop was studied for three types of micromixers. When comparing results, we found promising results in Reynolds numbers less than 0.1 for every style. Crucial differences were found between them when the degree of connections being twisted had increased, especially at 1.5 revolutions. The mixing index was greater than $90 \%$ independent of the Reynolds numbers and all ratios. Additionally, a drop of pressure was achieved along with the mixer of the twisted type 1.5 rev. higher than other mixers.
\end{abstract}

Keywords: Computational fluid dynamic, Mixing Index, Passive Micromixer, Spilt, and recombination, Twisted.

\section{Introduction}

The microfluidic field is an indispensable and vital field for humanity's service and has wide applications in many life areas. Some of these areas include laboratory analysis of samples, detection of diseases and viruses. The most important devices in this field are ( $\mu$ - TAS) and lab-on-a-chip., [1-4], DNA and protein analysis, drug detection, as well as in industries, such as food industries, water filtration, and biochemical chemical analysis [5-9]. This area has seen rapid development and an increasing interest in the importance of the real threats facing society in health, prevention, and biosecurity. Such devices have experienced impressive growth. They have achieved higher reliability due to savings in effort and time, reduced size, more significant cost savings [10-11], multitasking, and lower error rates than in previous years. The focus was on microfluidic systems' components, especially on micromixers, which are the essential components. However, micromixers classify into two basic types, namely passive and active micromixers [12-13]. Micromixers that use energy from an external source, such as electricity, magnetic, acoustic, ultrasonic, microwaves [1418], are called active micromixers, whereas mixers without any energy are called passive micromixers, which and depend on one strategy, which is to treat walls of mixers in line with the highest efficiency for these mixers [19-25].

With regard to passive micromixers and to make better use of designs, researchers used many methods to manipulate the walls of the geometric shapes of mixers to produce the best designs in a manner that guarantees high efficiency and its production in the smallest possible space. Some of these mixers include curves, zigzags, obstacles, grooves, convergentdivergent, spilt-recombination, or mixing of these types with each other [26-30]. The mixing process in mixers is mainly based on the chaotic mixing to move fluids and forced them to mix over a short path and in a small amount of time. One of these methods includes splitting and recombining fluids in several units with low to even high Reynolds numbers [31-33]. One of these designs has been used the baffles [34], another took an unbalanced form [35], and the other section combines different engineering shapes to achieve splitting and recombination [36]. The main idea of designing SAR mixers is to cut and dismantle liquids and reassemble them more than once into units and take advantage of the occurring vortices and layers that suffer from instability to be rearranged within the consecutive units of the mixer to produce a more homogeneous mixture. This method attracts many researchers who provide more reliable designs to serve the mixing 
process. This research is an attempt with ongoing efforts to achieve a design that contributes to a typical mixing process as much as possible.

This study aims to design a new passive micromixer model that works at a low Reynolds number and improves the mixing process. We use the SAR concept, namely spilt and recombination, in three dimensions to achieve this task. The parameters to test the effectiveness of the micromixer performance are listed below:

\# The basic model.

* Obstacles tested in three levels with comparisons.

* The twisted connections were tested with three levels with comparisons.

* Three ratios of velocity are used with comparisons.

\# The drop of pressure was tested with comparisons.

Fig. 1 shows the basic micromixer model with details and the scheme with all dimensions
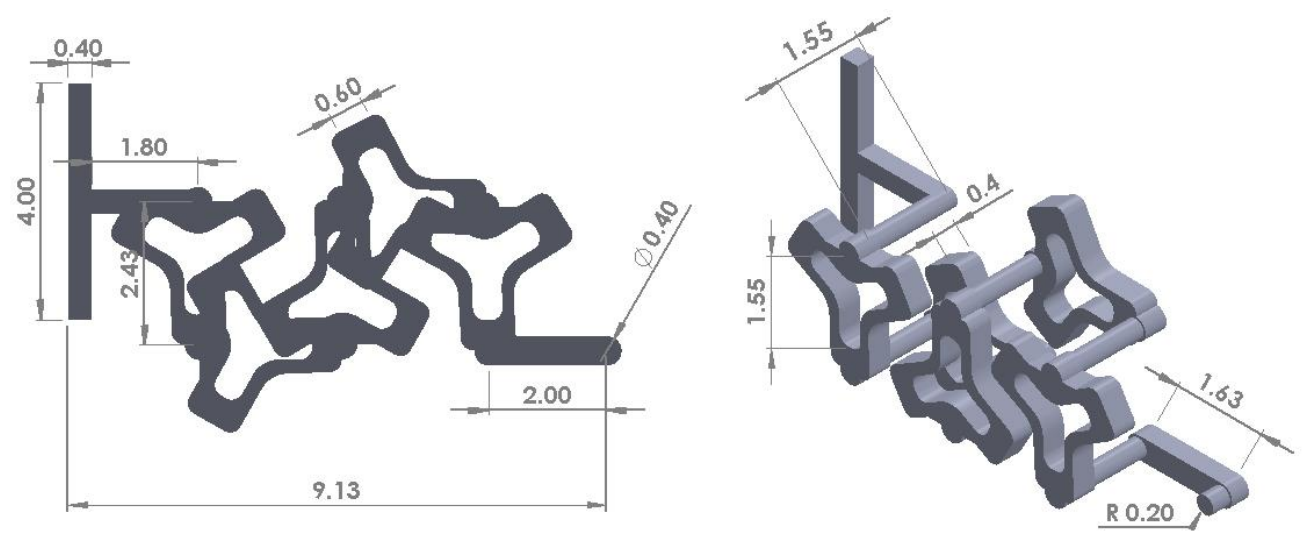

Fig. 1. Details of basic design in mm.

Fig. 2a illustrates the obstacle type with components representing the mixing's central unit. Three types of obstacles are utilized in this shape $(0.15,0.2,0.25) \mathrm{mm}$ to know obstacles' effect on the mixing efficiency. Other components remain the same (inlet's-connections-outlet ). Fig . $2 b$ shows the third type, a micromixer with twisted connections. The other elements are left except for the links that vary from three levels (1rev, 1.25rev, 1.5rev . ) to know the effective range of the micromixer's design.
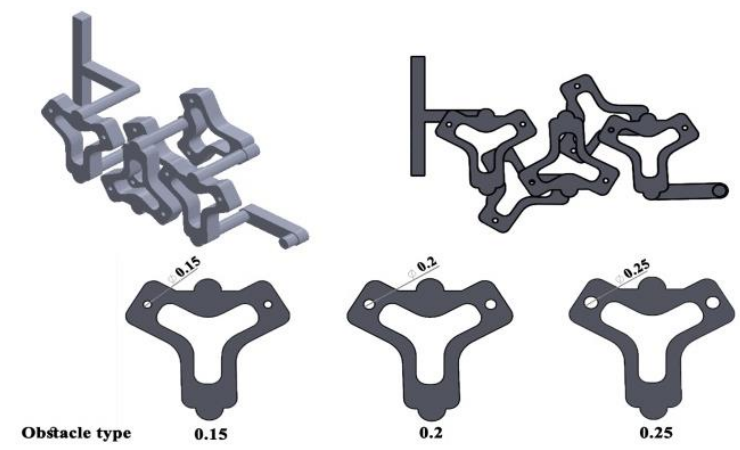

(a)

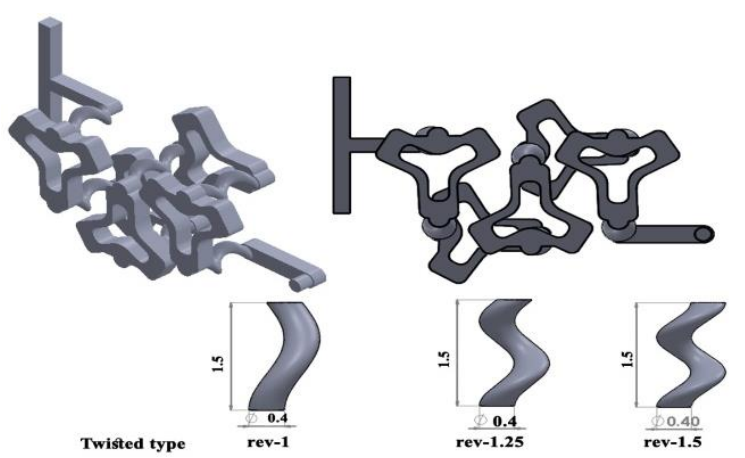

(b)

Fig. 2. (a) Obstacles types $(0.15,0.2,0.25) \mathrm{mm}$, (b) Twisted type with three types of connection. 


\subsection{Micromixer Model}

The type splitting and recombination micromixer has been used with added obstacles with different diameters and manipulated the connections straightness between the central units in the present study-all micromixer details as shown in Fig.1. The three-dimensional mixer's basic features show that the mixer is a T-type with two inlets with cross-sections of $(0.4 \times 0.4) \mathrm{mm}$ and a circular outlet of $0.4 \mathrm{~mm}$. The inlet length is $1.8 \mathrm{~mm}$, and the outlet length is $2 \mathrm{~mm}$. The micromixer consists of five main units; each unit has had three wings. The connections between them are cylindrical with a length of $1.5 \mathrm{~mm}$ and a diameter of $0.4 \mathrm{~mm}$. The total length of the micromixer reaches $9.38 \mathrm{~mm}$. Ethanol into inlet 1 and inlet 2 represents the water. The properties are listed in Tab.1.

Table 1. Properties of fluids at $20^{\circ} \mathrm{C}$

\begin{tabular}{|l|l|l|l|}
\hline Fluid & $\begin{array}{l}\text { Density } \\
\left(\mathrm{kg} \mathrm{m}^{-3}\right)\end{array}$ & $\begin{array}{l}\text { Viscosity } \\
\left(\mathrm{kgm}^{-1} \mathrm{~s}^{-1}\right)\end{array}$ & $\begin{array}{l}\text { Diffusivity } \\
\left(\mathrm{m}^{2} \mathrm{~s}^{-1}\right)\end{array}$ \\
\hline Water & $9.998 \times 10^{2}$ & $0.9 \times 10^{-3}$ & $1.2 \times 10^{-9}$ \\
\hline Ethanol & $7.890 \times 10^{2}$ & $1.2 \times 10^{-3}$ & $1.2 \times 10^{-9}$ \\
\hline
\end{tabular}

\subsection{Numerical Study}

To perform this study, ANSYS Fluent R19 was used to evaluate the micromixer's performance, with two inlets, inlet-1 for ethanol and inlet-2 for water. The properties are listed in Table. 1. Reynolds numbers were used as a criterion of performance. These were calculated according to Equation (1). The study takes into consideration steady-state, Newtonian, and incompressible conditions being assumed to solve the governing equations in addition to the convection and diffusion equation for the mass transport, which the equations are respectively shown in Equation (2) and Equation (3), and Equation (4). Gravity and body forces were ignored.

$$
\begin{gathered}
\operatorname{Re}=\frac{\rho v d}{\mu} \\
\rho \frac{\partial V}{\partial t}+\rho V \cdot \nabla V=-\nabla P+\mu \nabla^{2} V \\
\frac{\partial \rho}{\partial t}+\nabla \cdot(\rho V)=\mathrm{O} \\
\frac{\partial c}{\partial t}+V \cdot \nabla c=D \nabla^{2} c
\end{gathered}
$$

To avoid the errors, a high-quality mesh was used as a cut-cell Cartesian (hexahedral cells), valid for the complex geometry (Computational Fluid Dynamics) in the simulation process. The steady solver with the SIMPLEC algorithm is assumed to achieve coupling between pressure and velocity. The second-order upwind scheme uses to discretize the convective term of the governing equations. This method also decreases any false diffusion, which could otherwise be unsuitable in mixing problems. Solutions are considered to be converged when each variable's relative residual is less than 10-5. The meshing was tested at three levels of the grid to select the favorable one. After the grid analysis, simulations performed using the number of approximately $(9.5 \times 105,6.5 \times 105,2.5 \times 105)$ by grid convergence index methodology. the study chose $9.5 \times 10^{5}$ in all simulation activities, where was suitable according to the GCI ( Grid Convergence Index ) calculations.

\subsection{Mixing Efficiency Evaluation}

Efficiency can be calculated according to Eq. (5) as shown below, where 0 refers to no mixing and 1 means that the mixing is completing.

$$
\mathrm{MI}=1-\frac{\sqrt{1 / N \sum_{1}^{N}\left(C-C^{*}\right)^{2}}}{\sqrt{1 / N \sum_{1}^{N}\left(C^{o}-C^{*}\right)^{2}}}
$$


Here $N$ is the number of points in the section selected to test the mixing index. $\mathrm{C}$ is the scaled concentration value at that point, while $\mathrm{C}^{\circ}=0$ due to no mixing and $\mathrm{C}^{*}=1$ if the mixing was perfect, that meaning the result still a constant denominator value of 0.5 in Eq. (5).

\subsection{Grid Independence Test}

The present study introduced A grid-independent analysis to select the micromixer's mesh resolution. The study applied the GCI method at the mixer outlet for three levels [37-38]. To achieve these steps, it would use Eqs. (6)-(7) :

$$
e_{a}^{21}=\left|\frac{\phi_{1}-\phi_{2}}{\phi_{1}}\right|
$$

The variable $e_{a}^{21}$ approximate relative error with the symboles1, 2, and 3 indicates the mesh's three levels. Namely fine, medium, and coarse, and $\phi$ is used to express the mixing index (M) in the calculations.

$$
G C I_{21}=\frac{1.25 e_{a}^{21}}{\left(r_{21}^{p}-1\right)}
$$

Here 1.25 is the safety factor, and $r_{21}=h_{2} / h_{1}$ is the grid refinement factor. The result was $\mathrm{GCI}_{21}=0.14$, as shown in Tab. (2). The study selected a $9.5 \times 10^{5}$ grid system to perform the simulation calculation. Occasionally, by increasing the number of cells, it is not essential to improve the grid's accuracy, as shown in Fig. 3, where there is no significant efficiency improvement relative to the number of cells.

Table 2. Results of calculations of Grid independence

\begin{tabular}{|l|c|c|}
\hline Parameter & Symbols & Value \\
\hline Number of cells & $\mathrm{N} 1 / \mathrm{N} 2 / \mathrm{N} 3$ & $9.5^{*} 10^{5} / 6.5^{*} 10^{5} / 2.5^{*} 10^{5}$ \\
\hline Apparent order & $\mathrm{P}$ & 1.3 \\
\hline Extrapolated values & $\phi_{\text {ext }}^{21}$ & 0.52 \\
& $\phi_{\text {ext }}^{32}$ & 0.5 \\
\hline Approximate relative error & $e_{a}^{21}$ & 0.077 \\
& $e_{a}^{32}$ & \\
\hline Extrapolated relative & $e_{\text {ext }}^{21}$ & 0.141 \\
& $e_{\text {ext }}^{32}$ & 0.12 \\
\hline Grid convergence index & $\mathrm{GCI}_{21}$ & 0.256 \\
& $\mathrm{GCI}_{32}$ & 0.14 \\
& & 0.26 \\
\hline
\end{tabular}

\subsection{Mesh Selection}

The methodology stated above in section 1.4 (GCI) was applied to select the most suitable mesh for all simulation processes. After the practical experiments, there was no need to increase the number of cells where the results were similar, as shown in Fig. 3. 


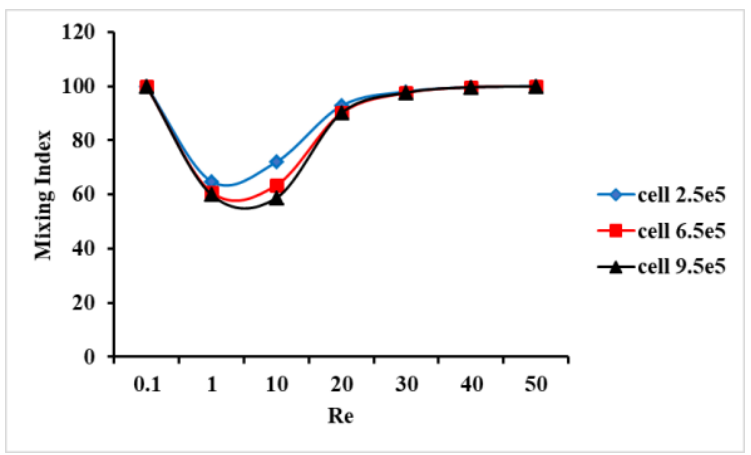

Fig. 3: Analysis of grids for the basic model.

\section{Results and Discussion}

This section of the work shows an evaluation of the present study with the experimental and numerical study of Victorov et al. (2015). The validation shows a considerable convergence between the present numerical analysis with the investigation as mentioned earlier; according to Fig. 4 between the two studies and the current study, it was found that the deviation from the numerical and experimental studies is. $0.18 \%$ and $0.28 \%$, respectively which is expected because of the nature of the experimental setup and conditions that are reflected in the numerical study. These satisfying results give more confidence and support to the present study.

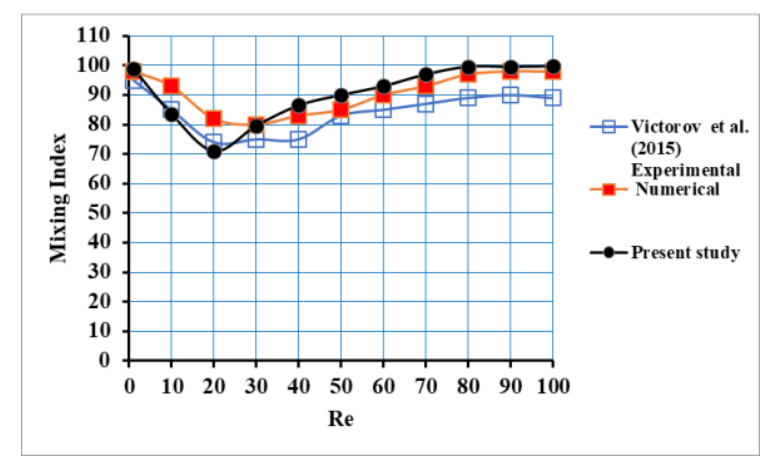

Fig. 4: Validation of present study with experimental and numerical Victorov et al. (2015) study.

In this work, the winged mixer was tested for three types of design the basic type, the type that contains an obstacle to three diameters $(0.15-0.02-0.25)$, and the type which has twisted connections between the basic units and for three cases (1rev - 1.25rev - 1.5rev ). In addition to the test of the inlet's velocity ratio to three levels (1-2- 3 ). These types were mainly tested for a wide range of Reynolds number $0.1 \geq \operatorname{Re} \geq 100$. The best performance was selected for each of the three cases, and then the best three types were compared together. The main objective of this study was to choose the best performance for these types

According to Fig. 5, the available data shows how the three models' comparison was made. The results showed that the model with twisted connections with a degree of 1.5 revs. For all Reynolds numbers and all inlet velocities, ratios were the best. See (Fig. 5a,b,c). 


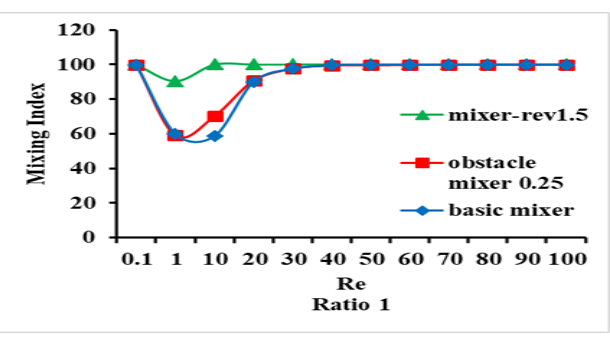

(a)

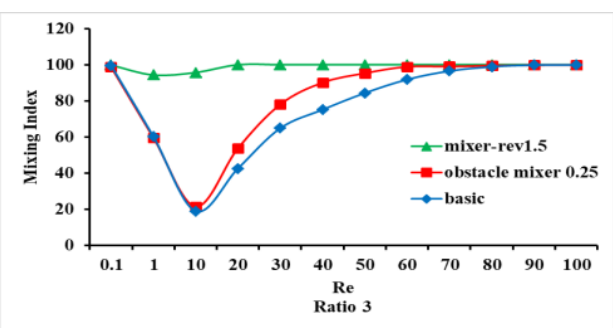

(c)

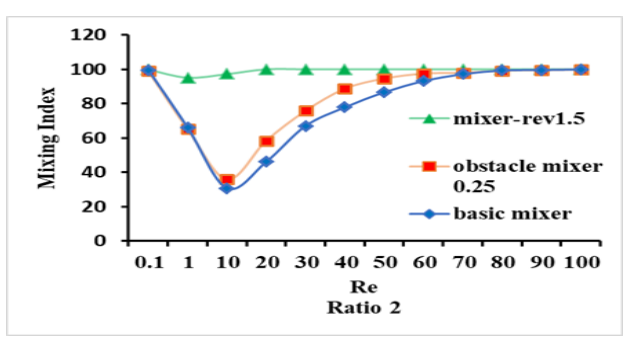

(b)

Fig. 5. Results of comparison three ratios, (a) ratio1, (b) ratio 2, (c) ratio 3.

Here, Fig. 6 shows the three types' performance (fundamental, obstacle 0.25, twisted 1.5rev) for three ratios. Comparisons were made at $\mathrm{Re}=1,10$, and 30; the figure shows by the mass fraction the differences range between them, where the type twisted 1.5rev. was the best in all stages of the simulation activities, thanks to the twisted connections that contribute to accelerating the layers stirring and chaotic the flow.

Also, Fig. 7 shows the three types' performance of the mixers at $\mathrm{Re}=10$. The twisted type is the best between them again by distributing the concentration in the mixing units, which gives apparent consideration to the twisted type is the best.

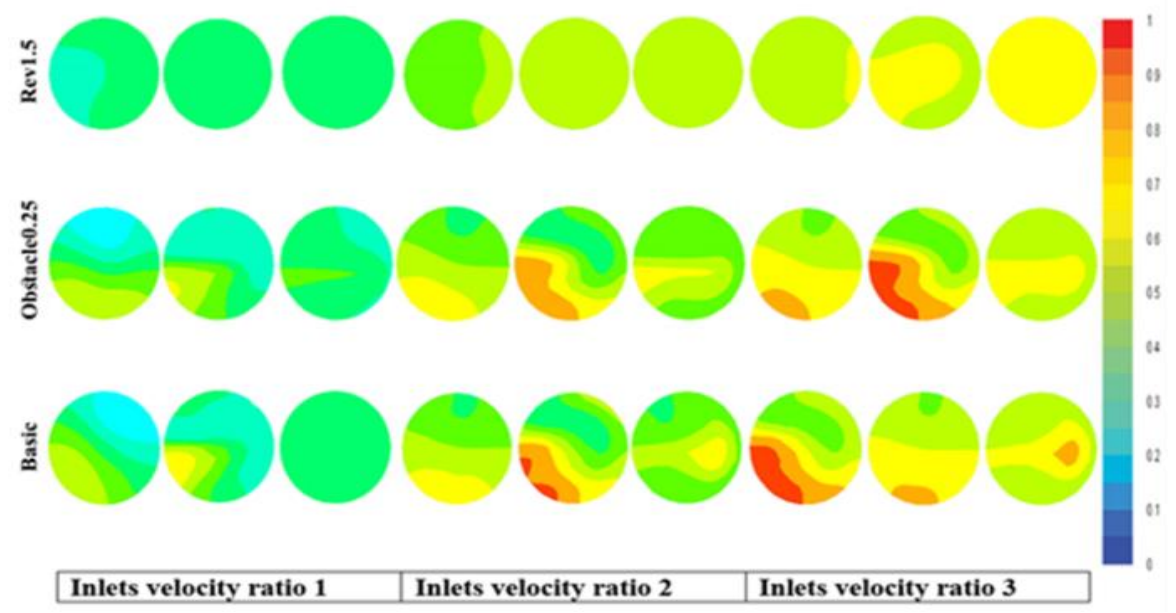

Fig. 6: Results of mixing for three ratios for three types at $\mathrm{Re}=1,10$ and 30 at the exit.

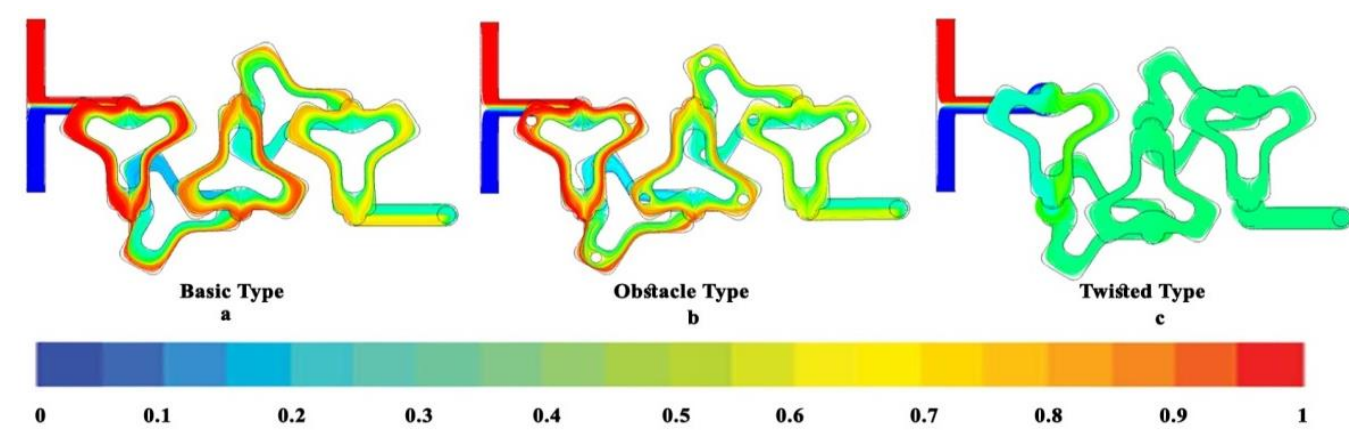

Fig. 7: Distribution of concentration according to mass fraction for three types (a) basic type (b) obstacle type (c) twisted type. 
Finally, the microchannel performance study's necessary factor should be the drop pressure behavior within the test stages. It is essential to evaluate the consumption of power as to whether the design is suitable for the energy side.

(Fig. 8) shows the relationship between the Reynolds number and pressure drop. The pressure drop develops linearly with increasing Reynolds number and is largely achieved with the twisted connections than the other shapes.

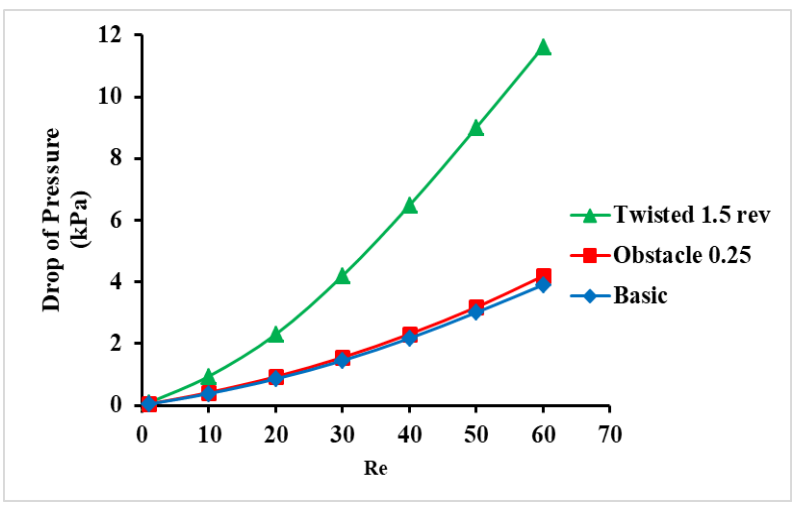

Fig. 8: Drop of pressure for three types (basic- obstacle 0.25-twisted rev.1.5) with Re.

\section{Conclusion}

A 3D passive micromixer with five repeat units as wing shape was proposed. This work on a splitting and recombination concept by dividing the fluids, stirring, and rotating them to produce good results in the smallest space possible depending on the micromixer's shape. The examination was made numerically with a wide range of Reynolds numbers $0.1 \geq \mathrm{Re} \geq 100$ for three types of micromixer (basic-with obstacle-with twisted connections ) and changing the inlet's velocity ratio. A comparison between them is performing. The results showed good performance for all with an increase in Reynolds number. However, the worst performance occurred over $1 \geq \operatorname{Re} \geq 10$ except the twisted type with 1.5 revs. gave good results for all cases. When comparisons were made with three models, the obstacle type (0.15-0.2-0.25 ) $\mathrm{mm}$ and the basic model, no change in efficiency could be observed. However, when comparisons were made using the twisted connections model (1 revs-1.25 revs-1.5 revs ), the results were superior with the twisted type with1.5 revs- the model producing the best results of all the tests where the lowest efficiency was $90 \%$. The type mentioned previously showed an increase in the drop of pressure in all of them, supporting the design and giving it a priority to study and importance and lower costs in power required to operate it. The total length of the micromixer reached $9.38 \mathrm{~mm}$. It was observed that the mixing was completed in the third unit with a length of $4.65 \mathrm{~mm}$, which means we can decrease the size to be suitable with the manufacturing requirement at less than the number of mixing units. Finally, the proposed micromixer is ideal for high mixing efficiency. Moreover, it is not affected by the inlet velocity ratio, and it achieves an increase in the pressure drop; hence it can be used in the industrial field. In the future, it would be beneficial to conduct a practical and experimental study on the proposed mixer to match it with the numerical analysis study.

\section{References}

[1] H.A. Stone, A.D. Stroock, A. Ajdari, "Engineering flows in small devices: microfluidics toward a lab-on-a-chip,". Annu. Rev. Fluid Mech., vol. 36, pp. 381-411, 2004.

[2] A.M. Streets, \& Y. Huang, Biomicrofluidics. Chip in a lab: "Microfluidics for next-generation life science research.,". Biomicrofluidics, vol. 7, no. 1, pp. 011302, 2013.

[3] L.L. Lazarus, C.T. Riche, B.C. Marin, M. Gupta, N. Malmstadt, R.L. Brutchey. "Two-phase microfluidic droplet flows of ionic liquids for the synthesis of gold and silver nanoparticles,". ACS applied materials \&interfaces, vol.no.6, pp.3077-3083, 2012. 
[4] Q. Tseng, I. Wang, E. Duchemin-Pelletier, A. Azioune, N.Carpi, J.Gao O. Filhol, M. Piel, M.Théry, M. Balland. "A new micropatterning method of soft substrates reveals that different tumorigenic signals can promote or reduce cell contraction levels,". Lab Chip., vol. 11, no. 13, pp. 2231-2240, 2011.

[5] G. Jia, K.-S. Ma, J. Kim, J.V. Zoval, R. Peytavi, M.G. Bergeron,\& M.J. Madou. "Dynamic automated DNA hybridization on a CD (compact disc) fluidic platform,". Sens. Actuators B. vol. 114, no.1: pp. 173-181, 2006.

[6] S.Hirschberg, R. Koubek, F. Moser, \& J. Schöck,. An improvement of the Sulzer SMX ${ }^{\mathrm{TM}}$ static mixer significantly reducing the pressure drop,". Chem. Eng. Res. Des., vol. 87, no. 4: pp. 524-532, 2009.

[7] A. Alam, A. Afzal, \& K. Y. Kim. "Mixing performance of a planar micromixer with circular obstructions in a curved microchannel,". Chem. Eng. Res. Des. vol. 92, no. 3, pp. 423-434, 2014.

[8] S. Hossain, A. Husain, \& K. Kim. "Optimization of micromixer with staggered herringbone grooves on top and bottom walls. Engineering Applications of computational,". Fluid Mechanics, vol. 5, no. 4, pp. 506-516, 2011.

[9] V. Srinivasan, V.K. Pamula, R.B. Fair. "An integrated digital microfluidic lab-on-a-chip for clinical diagnostics on human physiological fluids,". Lab Chip., vol. 4, no. 4, pp.310-315, 2004.

[10] G.S. Jeong, S. Chung, C.B. Kim,. "Applications of micromixing technology,". Analyst, vol. 135, no. 3, pp. 460-473, 2010.

[11] N. T. Nguyen, \& X. Huang. Mixing in microchannels based on hydrodynamic focusing and time-interleave segmentation: modeling and experiment. Lab Chip., vol. 5, no. 11: (2005) pp.1320-1326.

[12] R.B. Fair, "Digital microfluidics: is a true lab-on-a-chip possible?,". Microfluid. Nanofluid., vol. 3, no. 3, pp. 245281, 2007.

[13] D.R. Meldrum, M.R. Holl. "Microscale bioanalytical systems,". Science ., vol. 297, no. 5584. pp. 1197-1198, 2002.

[14] I Meisel, P. Ehrhard. "Electrically-Excited (Electroosmotic) Flows in Microchannels for Mixing Applications,". Europ J. Mech: B-Fluid ., vol. 25, no. 4, pp. 491-504, 2006.

[15] D. Nouri, A. Zabihi-Hesari, \& M. Passandideh-Fard. "Rapid mixing in micromixers using a magnetic field,". Sens. Actuators, A., vol. 255, pp. 79-86, 2017.

[16] Luong, T. D., Phan, V. N., \& Nguyen, N. T. "High-throughput micromixers based on acoustic streaming induced by a surface acoustic wave,". Microfluid. Nanofluid., vol. 10, no. 3, pp. 619-625,2001.

[17] Z. Yang, S. Matsumoto, H. Goto, M. Matsumotob, R. Maeda. "Ultrasonic micromixer for microfluidic systems,". Sen. Actuators, A., vol. 93, no. 3, pp. 266-272, 2001.

[18] R. Cecilia, U. Kunz, T. Turek. "Possibilities of process intensification using microwaves applied to catalytic,". microreactors. Chem Eng Process, vol. 46, no. 9, pp. 870-81, 2007.

[19] Y. Lin, "Numerical characterization of simple three-dimensional chaotic micromixers,". Chem. Eng. J., vol. 277: (2015) pp. 303-311.

[20] P. S. Fodor, M. Itomlenskis, \&M. Kaufman, "Assessment of mixing in passive microchannels with fractal surface patterning,". The European Physical Journal-Applied Physics, vol. 47, no. 3, pp. 31301, 2009.

[21] J.I. Martínez-López, M. Mojica, C.A. Rodríguez, H.R. Siller. "Xurography as a rapid fabrication alternative for pointof-care devices: Assessment of passive micromixers,". Sensors . vol. 16, no. 5, pp. 1-21, 2016.

[22] H. SadAbadi, M. Packirisamy, \& R. Wüthrich. "High performance cascaded PDMS micromixer based on split-andrecombination flows for lab-on-a-chip applications". RSC Advances, vol. 3, no. 20, pp.7296-7305, 2013.

[23] J.T. Yang, K.J. Huang, Y.C. Lin. "Geometric effects on fluid mixing in passive grooved micromixers". Lab a Chip., vol. 5, no. 10, pp.1140-1147, 2005.

[24] X. Chen, \& Li. "A novel passive micromixer designed by applying an optimization algorithm the zigzag microchannel,". Chem. Eng. J., vol. 313, pp. 1406-1414, 2017.

[25] X. Chen, Z. Zhao. Numerical investigation on layout optimization of obstacles in a three-dimensional passive micromixer. Anal.Chim. Acta., vol. 964: (2017) pp.142-149.

[26] A. Alam, \& K. Y. Kim. "Analysis of mixing in a curved microchannel with rectangular grooves," Chem.Eng.J., vol. 181, pp.708-716, 2012. 
[27] A. Afzal, \& K. Y. Kim. "Passive split and recombination micromixer with convergent-divergent walls,". Chem. Eng. J., vol. 203, pp. 182-192, 2012.

[28] W. Raza, S. Hossain, \& K. Y. Kim. "Effective mixing in a short serpentine split-and-recombination micromixer,". Sens. Actuators B Chem., vol. 258, pp. 381-392, 2018.

[29] Viktorov, V., Mahmud, M. R., \& Visconte, C. (2015). Comparative analysis of passive micromixers at a wide range of Reynolds numbers. Micromachines, vol. 6, no. 8, pp.1166-1179.

[30] T. Tofteberg, M. Skolimowski, E. Andreassen, \& O. Geschke. A novel passive micromixer: lamination in a planar channel system. .Microfluid. Nanofluid., vol. 8, no. 2: (2010) pp. 209-215.

[31] H. Chen, \& J. C. Meiners. "Topologic mixing on a microfluidic chip," Appl. Phys. Lett., vol. 84, no. 12, pp.2193 $2195,2004$.

[32] S. Hossain, \& K. Y. Kim. "Mixing analysis in a three-dimensional serpentine split-and-recombine micromixer,". Chem. Eng. Res. Des., vol. 100, pp. 95-103, 2015.

[33] A. Alam, K. Y. Kim. "Mixing performance of a planar micromixer with circular chambers and crossing constriction channels. Sens,". Actuators, B., vol. 176, pp. 639-652, 2013.

[34] W. Raza, K. Y. Kim. "Asymmetrical Split-and-Recombine Micromixer with Baffles," Micromachines., vol. 10, no. 12, pp. 844-859, 2019.

[35] M. A. Ansari, K. Y. Kim. "Mixing performance of unbalanced split and recombine micromixers with circular and rhombic sub-channels,". Chem. Eng. J., vol. 162, no. 2, pp. 760-767, 2010.

[36] I. Shah, S. W. Kim, K. Kim, Y. H. Doh, \& K. H. Choi. "Experimental and numerical analysis of Y-shaped split and recombination micro-mixer with different mixing units,".Chem. Eng. J., vol. 385: pp. 691-706, 2018.

[37] M.S.M. Ali, C.J. Doolan, V. Wheatley. "Grid convergence study for a two-dimensional simulation of flow around a square cylinder at a low Reynolds number,". pp. 1-6, 2009.

[38] I. Celik, U. Ghia, P. R. C. Freitas, H. Coleman, P. Raad, J. "Procedure for estimation and reporting of uncertainty due to discretization in CFD applications,". Fluids Eng., vol. 130, no. 7: (2008) pp.1-4. 